\title{
Superior cavopulmonary anastomosis timing and outcomes in infants with single ventricle
}

\author{
James F. Cnota, MD, ${ }^{\mathrm{a}}$ Kerstin R. Allen, MA, ${ }^{\mathrm{b}}$ Steven Colan, MD, ${ }^{\mathrm{c}}$ Wesley Covitz, MD, ${ }^{\mathrm{d}}$ \\ Eric M. Graham, MD, ${ }^{\mathrm{e}}$ David A. Hehir, MD, ${ }^{\mathrm{f}}$ Jami C. Levine, MD, ${ }^{\mathrm{c}}$ Renee Margossian, MD, ${ }^{\mathrm{c}}$ \\ Brian W. McCrindle, MD, ${ }^{\mathrm{g}}$ L. LuAnn Minich, MD, ${ }^{\mathrm{h}}$ Shobha Natarajan, MD, ${ }^{\mathrm{i}}$ Marc E. Richmond, MD, ${ }^{\mathrm{j}}$ and \\ Daphne T. Hsu, MD, ${ }^{\mathrm{k}}$ for the Pediatric Heart Network Investigators
}

\begin{abstract}
Objectives: We sought to identify factors associated with the timing and surgical outcomes of the superior cavopulmonary anastomosis.

Methods: The Pediatric Heart Network's Infant Single Ventricle trial database identified participants who underwent superior cavopulmonary anastomosis. Factors potentially associated with age at superior cavopulmonary anastomosis, length of stay and death by 14 months of age were evaluated. Factors included subject demographics, cardiac anatomy, measures from neonatal hospitalization and pre-superior cavopulmonary anastomosis visit, adverse events, echocardiographic variables, intraoperative variables, superior cavopulmonary anastomosis type, and number of concurrent cardiac surgical procedures. Age at superior cavopulmonary anastomosis was analyzed using Cox proportional hazards regression. Natural log length of stay was analyzed by multiple linear regression.
\end{abstract}

Results: Superior cavopulmonary anastomosis was performed in 193 subjects at 5.2 months of age (interquartile range, $4.2,6.2$ ) and weight of $5.9 \mathrm{~kg}$ (interquartile range, 5.3, 6.6). The median length of stay was 7 days (interquartile range, 6,10 ). There were 3 deaths and 1 transplant during the superior cavopulmonary anastomosis hospitalization, and 3 deaths and 3 transplants between discharge and 14 months of age. Age at superior cavopulmonary anastomosis was associated with center and interstage adverse events. A longer length of stay was associated with younger age and greater case complexity. Superior cavopulmonary anastomosis type, valve regurgitation, ventricular ejection fraction, and ventricular end-diastolic pressure were not independently associated with age at superior cavopulmonary anastomosis or the length of stay.

Conclusions: Greater case complexity and more frequent interstage adverse events are associated with an earlier age at superior cavopulmonary anastomosis. Significant variation in age at superior cavopulmonary anastomosis among centers, independent of subject factors, highlights a lack of consensus regarding the optimal timing. Factors associated with length of stay could offer insights for improving presuperior cavopulmonary anastomosis care and surgical outcome. (J Thorac Cardiovasc Surg 2013;145:1288-96)

From the Heart Institute, ${ }^{\text {a }}$ Division of Cardiology, Cincinnati Children's Hospital Medical Center, Cincinnati, Ohio; New England Research Institutes, Inc, ${ }^{\mathrm{b}}$ Watertown, Mass; Children's Hospital Boston, ${ }^{\mathrm{c}}$ Boston, Mass; Wake Forest University, ${ }^{\mathrm{d}}$ Winston-Salem, NC; Medical University of South Carolina, ${ }^{\mathrm{e}}$ Charleston, SC; Medical College of Wisconsin, ${ }^{\mathrm{f}}$ Milwaukee, Wis; Hospital for Sick Children, ${ }^{\mathrm{g}}$ Toronto, Ontario, Canada; Primary Children's Medical Center, ${ }^{\text {h }}$ Salt Lake City, Utah; Children's Hospital of Philadelphia, ${ }^{\mathrm{i}}$ Philadelphia, Pa; Columbia University, ${ }^{\mathrm{j}}$ New York, NY; and Children's Hospital at Montefiore, ${ }^{\mathrm{k}}$ Bronx, NY.

This study was supported by U01 grants from the National Heart, Lung, and Blood Institute (grants HL068269, HL068270, HL068279, HL068281, HL068285, HL068292, HL068290, HL068288, HL085057) and the Food and Drug Administration Office of Orphan Products Development. Its contents are solely the responsibility of the authors and do not necessarily represent the official views of the National Heart, Lung, and Blood Institute or National Institutes of Health.

Disclosures: Authors have nothing to disclose with regard to commercial support.

A list of Infant Single Ventricle trial participants is included in Appendix 1.

Received for publication Dec 15, 2011; revisions received June 1, 2012; accepted for publication July 26, 2012; available ahead of print Aug 30, 2012.

Address for reprints: James F. Cnota, MD, Heart Institute, Division of Cardiology, Cincinnati Children's Hospital Medical Center, MLC 2003, 3333 Burnet Avenue,

Cincinnati, OH 45229-3039 (E-mail: james.cnota@cchmc.org).

0022-5223/\$36.00

Copyright (c) 2013 by The American Association for Thoracic Surgery

http://dx.doi.org/10.1016/j.jtcvs.2012.07.069
The vast majority of children with single ventricle physiology undergo superior cavopulmonary anastomosis (SCPA) as a part of staged palliation. The factors associated with surgical outcomes have been addressed predominantly by single-center studies with varying and often conflicting results. These studies have identified age, weight-for-age, pulmonary artery pressure, ventricular morphology, atrioventricular valve regurgitation, and cardiopulmonary bypass time as variables associated with SCPA outcomes. ${ }^{1-7}$ However, the optimal timing for the procedure remains a topic of debate and has varied by center, in part, owing to a lack of consistent associations across these studies. This practice variation across centers could also play a role in determining the outcomes. ${ }^{8-10}$

With detailed clinical data from a multicenter network, the Infant Single Ventricle trial database offered a unique opportunity to evaluate factors associated with the timing of SCPA and subsequent surgical outcomes in infants undergoing SCPA. The present study sought to identify factors associated with SCPA timing and surgical outcomes. 


\section{Abbreviations and Acronyms \\ LOS = length of stay \\ SCPA $=$ superior cavopulmonary anastomosis}

\section{METHODS}

From August 2003 through July 2008, the Pediatric Heart Network conducted a randomized, double-blind, placebo-controlled clinical trial comparing the effects of angiotensin-converting enzyme inhibitor with those of placebo on somatic growth in infants with a single ventricle (ClinicalTrials.gov identifier NCT00113087). Infants 45 days old or younger with single ventricle physiology, stable systemic and pulmonary blood flow, and planned SCPA were enrolled at 10 North American centers. They were followed up from enrollment until 14 months of age. No significant differences were found between the placebo and treatment groups. Detailed descriptions of the Infant Single Ventricle trial design and methods, including enrollment, drug allocation, follow-up, data analysis, and results have been previously published. ${ }^{11,12}$

The trial database was queried for all infants undergoing SCPA during the study period. Age at SCPA was the outcome for surgical timing. Death, heart transplantation, hospital length of stay (LOS), unanticipated surgical or cardiac catheterization interventions after SCPA, days on ventilator, and interval until last chest tube removal were the clinical outcomes of interest. Unanticipated interventions included all post-SCPA cardiac catheter interventions and additional cardiac surgeries that occurred before discharge, excluding chest closure. The number of unanticipated interventions was dichotomized as none or 1 or more; total days on ventilator as 2 days or less or more than 2 days; and interval until last chest tube removal as 5 days or less or more than 5 days after SCPA. These thresholds for total days on ventilator and days until last chest tube removal represented a consensus opinion of suboptimal outcomes by the authors but also approximated the greatest quartile of days (rounded to an even number). Those who died or underwent heart transplantation during the SCPA hospitalization were excluded from the analyses of SCPA hospital LOS, days on ventilator, and interval until last chest tube removal. Potentially associated factors were selected from subject demographics, cardiac anatomy, measures from the neonatal hospitalization and clinical assessments at the pre-SCPA visit, adverse events during the interstage period between discharge from the neonatal hospitalization and SCPA, type of SCPA performed, and number of concurrent cardiac surgical procedures at SCPA (see Appendix 2). Adverse events were classified as nonserious, moderately serious, and serious using the Common Terminology Criteria for Adverse Events, version 3.0. ${ }^{13}$ All serious adverse events were adjudicated by an independent physician who was unaware of the treatment group assignment. Ventricular mass and volume measured by a core laboratory, and mass/volume ratio are expressed as $z$ scores relative to the body surface area and age, respectively. ${ }^{14}$ Angiotensin-converting enzyme inhibitor use was determined by whether the subject was taking any form of angiotensin-converting enzyme inhibitor (either an assigned study drug or open label medication) at the preSCPA clinical visit. The brain natriuretic peptide level was obtained at the pre-SCPA clinic visit and was measured at a central laboratory.

\section{Statistical Analysis}

Descriptive statistics for subject and SCPA hospitalization characteristics are presented as the median and interquartile range for continuous measures with a skewed distribution, mean \pm standard deviation for other continuous measures, and frequency and percentage for categorical variables.

Age at SCPA was analyzed using Cox proportional hazards regression, which allowed for the introduction of interstage adverse events as timevarying covariates. The LOS was analyzed after natural $\log$ transformation using multivariate linear regression. The linearity of associations with LOS was explored using generalized additive models. Dichotomous clinical outcomes were analyzed using logistic regression with Firth's penalized likelihood applied when the center was included in the models. To identify any possible nonlinear associations with the dichotomous outcome and time-to-event outcome, tertiles, median cutoffs, and square root or natural $\log$ transformations of potential associated factors were evaluated in logistic regression and Cox proportional hazards modeling.

Variables were considered in the multivariate model selection when the univariate or center-adjusted association was significant at $P \leq .15$. All models were adjusted for center to account for any differences in clinical management strategies between centers that were not fully captured by the Infant Single Ventricle database. Stepwise selection methods were used to determine all final models; predictors with $P \leq .05$ remained in the model. The effects of mean pulmonary artery pressure $(\mathrm{n}=98)$ and transpulmonary gradient $(n=60)$ were explored in univariate and multivariate analysis but were not included in the final modeling because of the large amount of missing data. Missing values were not imputed. Statistical analyses were performed using SAS Statistical Software, version 9.2 (SAS Institute, Cary, NC).

\section{RESULTS}

\section{Subject and SCPA Hospitalization Characteristics}

Of the 230 subjects randomized in the trial, 193 (84\%) underwent SCPA; 5 randomized subjects did not undergo SCPA but remained in the trial. The remainder withdrew from the trial before SCPA because of death $(\mathrm{n}=18)$, family preference $(n=8)$, heart transplantation $(n=3)$, physician preference $(n=1)$, lost to follow-up $(n=1)$, and other $(n=1)$.

The details of the SCPA hospitalization overall and stratified by type of SCPA are listed in Table 1. With the exception of the proportion of subjects undergoing aortopulmonary shunt takedown, the subject characteristics did not significantly differ by the type of SCPA performed. A trend $(P=.06)$ was seen toward more arrhythmia in subjects undergoing Hemi-Fontan procedures.

\section{Deaths and Heart Transplantations After SCPA}

There were 3 deaths and 1 heart transplantation that occurred during the SCPA hospitalization. An additional 3 deaths and 3 heart transplantations occurred between SCPA hospital discharge and the final study visit at 14 months of age (Table 2). The incidence of death was 3\% and of heart transplantation was $2 \%$ by 14 months of age. Deaths occurred predominantly in subjects with morphologic right ventricles, although it should be noted that $72 \%$ of the cohort had a morphologic right ventricle. Those who died also generally had low weight-for-age $z$ scores, with scores ranging from -4.2 to -1.0 . The average weight-forage $z$ score among those who survived SCPA was -1.61 (95\% confidence interval, -1.77 to -1.45 ).

\section{Age at SCPA}

The median age at SCPA was 5.2 months (interquartile range, 4.2, 6.2). The Cox proportional hazards regression model $\left(n=169, R^{2}=0.43\right)$ identified independent associations of younger age at SCPA with the following factors: center, nonblack race, younger age at neonatal palliative surgery, performance of the Norwood procedure, time-related 


\begin{tabular}{|c|c|c|c|c|c|c|c|}
\hline \multirow[b]{2}{*}{ Characteristic } & \multirow[b]{2}{*}{$\begin{array}{c}\text { All subjects } \\
(\mathbf{n}=193)\end{array}$} & \multicolumn{5}{|c|}{ Type of SCPA } & \multirow[b]{2}{*}{$P$ value* } \\
\hline & & $\begin{array}{c}\text { BBDCPA } \\
(\mathbf{n}=\mathbf{2 8})\end{array}$ & $\begin{array}{c}\text { BDCPA } \\
(\mathbf{n}=\mathbf{1 3 4})\end{array}$ & $\begin{array}{l}\text { Hemi-Fontan } \\
\quad(\mathbf{n}=\mathbf{2 3})\end{array}$ & $\begin{array}{l}\text { Hemi-Fontan with } \\
\text { BCA }(\mathbf{n}=\mathbf{3})\end{array}$ & $\begin{array}{c}\text { Kawashima } \\
\quad(\mathbf{n}=\mathbf{5})\end{array}$ & \\
\hline \multicolumn{8}{|l|}{ Age at SCPA (mo) } \\
\hline Median & 5.2 & 5.4 & 5.1 & 5.6 & 5.0 & 6.7 & .36 \\
\hline IQR & $4.2,6.2$ & $4.6,6.9$ & $4.1,6.0$ & $4.1,6.0$ & $4.6,7.1$ & $5.3,7.5$ & \\
\hline Gender & & & & & & & .89 \\
\hline Male & $136(70)$ & $20(71)$ & $94(70)$ & $16(70)$ & $3(100)$ & $3(60)$ & \\
\hline Female & $57(30)$ & $8(29)$ & $40(30)$ & $7(30)$ & 0 & $2(40)$ & \\
\hline Race & & & & & & & .34 \\
\hline White & $153(80)$ & $19(68)$ & $110(83)$ & $18(78)$ & $3(100)$ & $3(60)$ & \\
\hline Black & $27(14)$ & $7(25)$ & $17(13)$ & $2(9)$ & 0 & $1(20)$ & \\
\hline Other & $12(6)$ & $2(7)$ & $6(5)$ & $3(13)$ & 0 & $1(20)$ & \\
\hline Ventricular morphology & & & & & & & .027 \\
\hline Left & $34(18)$ & $3(11)$ & $26(19)$ & $5(22)$ & 0 & 0 & \\
\hline Right & $138(72)$ & $17(61)$ & $97(72)$ & $18(78)$ & $3(100)$ & $3(60)$ & \\
\hline Mixed & $21(11)$ & $8(29)$ & $11(8)$ & 0 & 0 & $2(40)$ & \\
\hline Concurrent cardiac surgical procedures (n) & & & & & & & .50 \\
\hline Median & 1.0 & 2.0 & 1.0 & 1.0 & 1.0 & 1.0 & \\
\hline IQR & 1,2 & 1,2 & 1,2 & 0,2 & 1,2 & 0,1 & \\
\hline \multicolumn{8}{|l|}{ Surgical procedures $(n) \dagger$} \\
\hline Atrial septectomy & $20(10)$ & $3(11)$ & $12(9)$ & $5(22)$ & 0 & 0 & .43 \\
\hline Patch repair of pulmonary artery stenosis & $71(37)$ & $9(32)$ & $47(35)$ & $11(48)$ & $1(33)$ & $3(60)$ & .55 \\
\hline Takedown of aortopulmonary shunt & $30(16)$ & $10(36)$ & $18(13)$ & $1(4)$ & $1(33)$ & 0 & .015 \\
\hline Takedown of RV-PA conduit & $45(23)$ & $5(18)$ & $34(25)$ & $3(13)$ & $2(67)$ & $1(20)$ & .25 \\
\hline Hospital LOS (d) & & & & & & & .12 \\
\hline Median & 7 & 8.5 & 7.0 & 8.0 & 8.0 & 8 & \\
\hline IQR & 6,10 & 6,13 & 6,9 & 7,11 & 6,12 & 7,8 & \\
\hline ICU LOS (d) & & & & & & & .22 \\
\hline Median & 4.0 & 5.0 & 4.0 & 4.0 & 3.0 & 4.0 & \\
\hline IQR & 3,5 & 3,7 & 3,5 & 3,5 & 2,8 & 4,6 & \\
\hline Ventilator support duration (d) & & & & & & & .79 \\
\hline Median & 2.0 & 2.0 & 2.0 & 2.0 & 2.0 & 2.0 & \\
\hline IQR & 1,2 & 1,3 & 1,2 & 1,2 & 1,2 & 1,2 & \\
\hline Interval to last chest tube removed (d) & & & & & & & .09 \\
\hline Median & 3.0 & 4.0 & 3.0 & 4.0 & 5.0 & 4.0 & \\
\hline IQR & 2,4 & 2,5 & 2,4 & 3,4 & 5,5 & 1,4 & \\
\hline $\begin{array}{l}\text { Postoperative arrhythmia requiring } \\
\text { intervention }\end{array}$ & $20(10)$ & $2(7)$ & $11(8)$ & $6(26)$ & $1(33)$ & 0 & .06 \\
\hline Bradycardia requiring artificial pacemaker $\ddagger$ & $13(62)$ & $2(100)$ & $7(58)$ & $4(67)$ & 0 & 0 & .57 \\
\hline Other arrhythmia $\ddagger$ & $4(19)$ & 0 & $3(25)$ & 0 & $1(100)$ & 0 & .16 \\
\hline \multicolumn{8}{|l|}{ Interventional cardiac catheterizations } \\
\hline 0 & $184(95)$ & $27(96)$ & $126(94)$ & $23(100)$ & $3(100)$ & $5(100)$ & \\
\hline 1 & $4(2)$ & 0 & $4(3)$ & 0 & 0 & 0 & \\
\hline$\geq 2$ & $5(3)$ & $1(4)$ & $4(3)$ & 0 & 0 & 0 & \\
\hline \multicolumn{8}{|l|}{ Postoperative surgery (minus chest closure) } \\
\hline 0 & $173(90)$ & $24(86)$ & $120(90)$ & $21(91)$ & $3(100)$ & $5(100)$ & \\
\hline 1 & $16(8)$ & $4(14)$ & $10(7)$ & $2(9)$ & 0 & 0 & \\
\hline$\geq 2$ & $4(2)$ & 0 & $4(3)$ & 0 & 0 & 0 & \\
\hline $\begin{array}{l}\text { Subjects discharged with supplemental } \\
\text { oxygen }\end{array}$ & $30(16)$ & $2(7)$ & $26(20)$ & $2(9)$ & 0 & 0 & \\
\hline Oxygen saturation at discharge $\S$ & $82.1 \pm 5.0$ & $81.7 \pm 4.7$ & $82.0 \pm 5.2$ & $82.5 \pm 4.5$ & $78.7 \pm 6.1$ & $85.8 \pm 3.7$ & \\
\hline Discharge medications & & & & & & & .29 \\
\hline Median & 4.0 & 3.5 & 4.0 & 4.0 & 2 & 4.0 & \\
\hline $\mathrm{IQR}$ & 3,5 & 3,5 & 3,5 & 2,5 & 2,2 & 3,4 & \\
\hline Any adverse event & $52(27)$ & $9(32)$ & $35(26)$ & $6(26)$ & $1(33)$ & $1(20)$ & .94 \\
\hline
\end{tabular}




\begin{tabular}{|c|c|c|c|c|c|c|c|}
\hline \multirow[b]{2}{*}{ Characteristic } & \multirow[b]{2}{*}{$\begin{array}{l}\text { All subjects } \\
(\mathbf{n}=193)\end{array}$} & \multicolumn{5}{|c|}{ Type of SCPA } & \multirow[b]{2}{*}{$P$ value* } \\
\hline & & $\begin{array}{c}\text { BBDCPA } \\
(\mathbf{n}=28)\end{array}$ & $\begin{array}{c}\text { BDCPA } \\
(n=134)\end{array}$ & $\begin{array}{c}\text { Hemi-Fontan } \\
(\mathbf{n}=\mathbf{2 3})\end{array}$ & $\begin{array}{c}\text { Hemi-Fontan with } \\
\text { BCA }(n=3)\end{array}$ & $\begin{array}{c}\text { Kawashima } \\
\quad(\mathbf{n}=\mathbf{5})\end{array}$ & \\
\hline \multicolumn{8}{|l|}{ Adverse event types $\dagger$} \\
\hline Cardiac general/arrhythmia & $17(9)$ & $2(7)$ & $12(9)$ & $1(4)$ & $1(33)$ & $1(20)$ & .32 \\
\hline Cardiac general & $9(5)$ & $1(4)$ & $6(4)$ & 0 & $1(33)$ & $1(20)$ & .08 \\
\hline Cardiac arrhythmia & $8(4)$ & $1(4)$ & $6(4)$ & $1(4)$ & 0 & 0 & 1.00 \\
\hline Infection & $17(9)$ & $6(21)$ & $9(7)$ & $2(9)$ & 0 & 0 & .17 \\
\hline Pulmonary/upper respiratory & $19(10)$ & $2(7)$ & $16(12)$ & $1(4)$ & 0 & 0 & .84 \\
\hline
\end{tabular}

Data presented as median and IQR, $\mathrm{n}(\%)$, mean \pm SD. Subjects who died or underwent heart transplantation before stage 2 hospital discharge were excluded from summary of hospital LOS, ICU LOS, total ventilator duration, and interval until last chest tube was removed. SCPA, Superior cavopulmonary anastomosis; $B B D C P A$, bilateral bidirectional cavopulmonary anastomoses; $B D C P A$, bidirectional cavopulmonary anastomosis; $B C A$, bilateral cavopulmonary anastomoses; $I Q R$, interquartile range; $R V$ - $P A$, right ventricularpulmonary artery; $L O S$, length of stay; ICU, intensive care unit. *P values assessed significance of difference between types of SCPA, using Kruskal-Wallace test for skewed continuous data, analysis of variance for other continuous data, and Fisher’s exact test for percentages. $\dagger$ Categories associated with $\geq 10 \%$ of subjects summarized. $\ddagger$ Percentage determined by subjects with arrhythmia. §Included only subjects who were not discharged with supplemental oxygen.

occurrence of interstage pulmonary or cardiac adverse events, and the presence of accessory pulmonary blood flow at pre-SCPA cardiac catheterization (Table 3).

\section{LOS After SCPA}

The median LOS was 7 days (interquartile range, 6, 10). After adjusting for center differences, the multivariate linear regression model of $\log \operatorname{LOS}\left(n=170, \mathrm{R}^{2}=0.33\right)$ identified independent associations of a longer LOS with the following factors: greater number of ventilator days during the neonatal palliative surgery, lower weight-for-age $z$ score at pre-SCPA clinic visit, greater end systolic volume $z$ score relative to body surface area on pre-SCPA echocardiogram, younger age at SCPA $(\log )$, and greater number of concurrent cardiac procedures at SCPA (Table 3).

\section{Occurrence of 1 or More Unanticipated Interventions During SCPA Hospitalization}

At least 1 unanticipated surgical or cardiac catheterization intervention occurred during SCPA hospitalization in $12 \%$ of our subjects. The types and frequencies of interventions are listed in Table 5. After adjusting for center, the multivariate logistic regression model $(\mathrm{n}=172, \mathrm{c}$-statistic $=0.86)$ identified that the occurrence of 1 or more unanticipated interventions was associated with a lower weight-forage $z$ score and higher ventricular mass $z$ score relative to the body surface area on the pre-SCPA echocardiogram (Table 4).

\section{Mechanical Ventilation for Longer Than 2 Days after SCPA}

Mechanical ventilation was used for longer than 2 days after SCPA in 23\% of subjects. After adjusting for center, the multivariate logistic regression model $(n=164$, c-statistic, 0.81) identified independent associations of mechanical ventilation for longer than 2 days with the following factors: younger than 140 days (lower tertile) of age at SCPA, brain natriuretic peptide greater than $135 \mathrm{pg} / \mathrm{mL}$, and a greater number of concurrent cardiac surgical procedures at SCPA (Table 5).

\section{Final Chest Tube Removed More Than 5 Days After SCPA}

The final chest tube was removed more than 5 days after SCPA in $14 \%$ of subjects. After adjusting for center, the multivariate logistic regression model $(\mathrm{n}=174$,

TABLE 2. Characteristics of subjects who died and subjects who underwent heart transplantation after SCPA

\begin{tabular}{|c|c|c|c|c|c|c|c|c|}
\hline $\begin{array}{c}\text { Interval to } \\
\text { outcome (d) }\end{array}$ & $\begin{array}{l}\text { ACE inhibitor use } \\
\text { at pre-SCPA visit }\end{array}$ & $\begin{array}{l}\text { Discharged } \\
\text { after SCPA }\end{array}$ & Gender & $\begin{array}{c}\text { Age at } \\
\text { SCPA (mo) }\end{array}$ & $\begin{array}{c}\text { Weight-for-age } \\
z \text { score } \\
\end{array}$ & $\begin{array}{c}\text { Ventricular } \\
\text { type }\end{array}$ & $\begin{array}{c}\text { Oxygen saturation } \\
\text { by pulse oximeter }(\%)\end{array}$ & $\begin{array}{c}\text { Pre-SCPA } \\
\text { BNP }(p g / m L)\end{array}$ \\
\hline \multicolumn{9}{|l|}{ Death } \\
\hline 1 & No & No & Male & 5.0 & -4.12 & Right & 70 & 801.0 \\
\hline 10 & Yes & No & Female & 4.9 & -1.37 & Right & 80 & 171.0 \\
\hline 66 & No & Yes & Female & 5.7 & -2.96 & Left & 67 & 1190.0 \\
\hline 95 & No & Yes & Male & 5.1 & -1.03 & Right & 72 & 141.0 \\
\hline 144 & No & No & Male & 3.4 & -2.23 & Right & 80 & \\
\hline 238 & No & Yes & Male & 8.2 & -2.69 & Right & 59 & \\
\hline \multicolumn{9}{|c|}{ Heart transplantation } \\
\hline 70 & Yes & No & Male & 3.0 & -1.73 & Right & 76 & 490.0 \\
\hline 94 & Yes & Yes & Female & 5.9 & -2.37 & Right & 70 & 135.0 \\
\hline 103 & No & Yes & Male & 3.3 & & Right & 65 & \\
\hline 121 & No & Yes & Male & 2.4 & -2.83 & Right & 71 & 30.5 \\
\hline
\end{tabular}

$S C P A$, Superior cavopulmonary connection; $A C E$, angiotensin-converting enzyme; $B N P$, brain natriuretic peptide. 
TABLE 3. Multivariate models of age at SCPA and SCPA hospital LOS

\begin{tabular}{|c|c|c|c|c|c|}
\hline Age at SCPA $(\mathrm{mo})\left(\mathrm{R}^{2}=0.43\right)(\mathrm{n}=169)$ & \multicolumn{2}{|c|}{ Age at SCPA (mo) } & $d f$ & HR $(95 \%$ CI $)$ & $P$ value \\
\hline Center & Refe & & 9 & Reference & $<.001$ \\
\hline \multicolumn{6}{|l|}{ Race } \\
\hline White & $5.18(4$ & & 2 & $1.93(1.2,3.1)$ & .005 \\
\hline Black & $6.03(4$ & & & Reference & \\
\hline Other & $4.52(3$ & & & $3.03(1.4,6.4)$ & \\
\hline Age at stage $1(d), \log$ transformation & $5.20(4$ & & 1 & $0.55(0.41,0.75)$ & $<.001$ \\
\hline \multicolumn{6}{|l|}{ Norwood procedure } \\
\hline Yes & $5.02(4$ & & 1 & $1.63(1.1,2.4)$ & .014 \\
\hline No & $5.64(5$ & & & Reference & \\
\hline \multicolumn{6}{|l|}{ Interstage pulmonary adverse event } \\
\hline Yes & $4.89(3$ & & 1 & $1.52(1.1,2.2)$ & .023 \\
\hline No & $5.43(4$ & & & Reference & \\
\hline \multicolumn{6}{|l|}{ Interstage cardiac adverse event } \\
\hline Yes & $4.74(3$ & & 1 & $2.31(1.5,3.5)$ & $<.001$ \\
\hline No & $5.31(4$ & & & Reference & \\
\hline \multicolumn{6}{|l|}{ Accessory sources of pulmonary blood flow before SCPA } \\
\hline Yes & $4.46(4$ & & 1 & $2.18(1.2,3.8)$ & .006 \\
\hline No & $5.28(4$ & & & Reference & \\
\hline \multicolumn{2}{|c|}{ SCPA hospital LOS $(d)$ natural $\log$ transformations $\left(R^{2}=0.33\right)(n=170)$} & Predictor & $d f$ & Slope $(95 \%$ CI $)$ & $P$ value \\
\hline \multicolumn{2}{|l|}{ Site } & Reference & 9 & Reference & .10 \\
\hline \multicolumn{2}{|l|}{ Ventilator duration during palliative surgery (d) (square root) } & $2.60 \pm 1.05$ & 1 & $0.13(0.05,0.21)$ & .003 \\
\hline \multicolumn{2}{|l|}{ Weight-for-age $z$ score before SCPA } & $-1.61 \pm 1.15$ & 1 & $-0.11(-0.19,-0.04)$ & .003 \\
\hline \multicolumn{2}{|l|}{ Pre-SCPA end-systolic volume BSA-adjusted $z$ score } & $3.37 \pm 3.79$ & 1 & $0.039(0.017,0.060)$ & $<.001$ \\
\hline \multicolumn{2}{|l|}{ Age at SCPA, log transformation (d) } & $5.07 \pm 0.30$ & 1 & $-0.49(-0.79,-0.18)$ & .002 \\
\hline \multicolumn{2}{|l|}{ Cardiac surgical procedures concurrent with SCPA (n) } & $1.47 \pm 1.12$ & 1 & $0.16(0.07,0.25)$ & $<.001$ \\
\hline
\end{tabular}

c-statistic $=0.84)$ identified an independent association of final chest tube removal at more than 5 days with a lower pre-SCPA ventricular ejection fraction (Table 4).

\section{DISCUSSION}

By assessing the factors associated with age at SCPA and measures of surgical outcome, the results of the present

TABLE 4. Multivariate models of post-SCPA unintended intervention, ventilator duration, and interval to chest tube removal

\begin{tabular}{|c|c|c|c|c|c|c|c|}
\hline$\underline{\text { Post-SCPA unintended intervention }(c-s t a t i s t i c}=\mathbf{0 . 8 6})$ & \multicolumn{2}{|c|}{ No intervention $(n=151)$} & \multicolumn{2}{|c|}{ Intervention $(\mathbf{n}=\mathbf{2 1})$} & \multirow{2}{*}{$\frac{d f}{9}$} & \multirow{2}{*}{$\frac{\text { OR }(95 \% \text { CI })}{\text { Reference }}$} & \multirow{2}{*}{$\frac{P \text { value }}{.37}$} \\
\hline Site & \multicolumn{2}{|c|}{ Reference } & \multicolumn{2}{|c|}{ Reference } & & & \\
\hline Weight-for-age $z$ score & \multicolumn{2}{|c|}{$-1.52 \pm 1.13$} & \multicolumn{2}{|c|}{$-2.38 \pm 1.13$} & 1 & $0.50(0.30-0.83)$ & .007 \\
\hline BSA-adjusted mass $z$ score, median cutoff & & 1 & & .013 \\
\hline$<3.94$ & \multicolumn{2}{|c|}{$83(95.4)$} & \multicolumn{2}{|c|}{$4(4.6)$} & \multicolumn{3}{|c|}{ Reference } \\
\hline$\geq 3.94$ & \multicolumn{2}{|c|}{$68(80.0)$} & \multicolumn{2}{|c|}{$17(20.0)$} & \multicolumn{3}{|c|}{$3.92(1.34-11.51)$} \\
\hline Total ventilator duration $($ c-statistic $=\mathbf{0 . 8 1})$ & $\leq 2 \mathrm{~d}(\mathrm{n}=126)$ & $>2$ d ( I & $=\mathbf{3 8})$ & $d f$ & & $\mathbf{R}(95 \% \mathbf{C I})$ & $P$ value \\
\hline Site & Reference & Refe & ence & 9 & & Reference & .48 \\
\hline BNP (pg/mL), upper tertile & & & & 1 & & & .005 \\
\hline$\leq 135$ & $91(82.0)$ & 20( & 8.0) & & & Reference & \\
\hline$>135$ & $35(66.0)$ & 18 & 4.0) & & 3.4 & $6(1.45-8.28)$ & \\
\hline Age at SCPA, lower tertile (d) & & & & 1 & & & .005 \\
\hline$>140$ & $93(81.6)$ & 21( & 8.4) & & & Reference & \\
\hline$\leq 140$ & $33(66.0)$ & 17 & 4.0) & & 3.5 & $9(1.46-8.85)$ & \\
\hline Concurrent cardiac surgical procedures (n) & $1.43 \pm 1.22$ & $1.77=$ & 0.96 & 1 & 1.9 & $2(1.23-3.02)$ & .005 \\
\hline$\underline{\text { Interval to chest tube removal }(\mathrm{c}-\mathrm{statistic}=\mathbf{0 . 8 4})}$ & $\leq 5 \mathrm{~d}(\mathrm{n}=148)$ & $>\mathbf{5}$ & $(\mathbf{n}=\mathbf{2 6})$ & $d f$ & & $\mathbf{R}(95 \% \mathbf{C I})$ & $P$ value \\
\hline Site & Reference & & ference & 9 & & Reference & .044 \\
\hline Pre-SCPA ejection fraction $(\%)$ & $58.29 \pm 9.44$ & 52. & $3 \pm 9.57$ & 1 & & $93(0.89-0.98)$ & .003 \\
\hline
\end{tabular}

ORs and slopes associated with continuous variables represent effect of 1-unit increase of continuous variable on outcome. SCPA, Superior cavopulmonary anastomosis; $O R$, odds ratio; $C I$, confidence interval; $B S A$, body surface area; $B N P$, brain natriuretic peptide. 
TABLE 5. Types of unanticipated postoperative procedures

\begin{tabular}{|c|c|}
\hline Category & $\mathbf{n}$ \\
\hline \multicolumn{2}{|l|}{ Cardiothoracic surgery } \\
\hline Thoracostomy tube & 11 \\
\hline Chest exploration & 4 \\
\hline Central or Blalock-Taussig shunt & 3 \\
\hline Device implantation & 2 \\
\hline Diaphragm plication & 2 \\
\hline Pulmonary artery plasty & 1 \\
\hline Shunt revision/thrombectomy & 1 \\
\hline Extracorporeal membrane oxygenator support & 1 \\
\hline Mediastinal debridement & 1 \\
\hline Right pneumonectomy & 1 \\
\hline Heart transplantation & 1 \\
\hline Ligation of left aortopulmonary collateral & 1 \\
\hline Coarctation repair & 1 \\
\hline Repair bleeding chest tube, evacuate hemothorax & 1 \\
\hline \multicolumn{2}{|l|}{ Cardiac catheterization } \\
\hline \multicolumn{2}{|l|}{ Angioplasty } \\
\hline Pulmonary artery & 4 \\
\hline Aorta & 2 \\
\hline Superior vena cava & 2 \\
\hline \multicolumn{2}{|l|}{ Stent } \\
\hline Superior vena cava & 1 \\
\hline Pulmonary artery & 1 \\
\hline \multicolumn{2}{|l|}{ Coil } \\
\hline Artery & 5 \\
\hline Vein & 1 \\
\hline Unspecified & 1 \\
\hline \multicolumn{2}{|l|}{ Noncardiac } \\
\hline Extremity amputation & 2 \\
\hline Extremity debridement & 2 \\
\hline Extremity skin graft & 1 \\
\hline Gastrostomy tube and Nissen fundoplication & 1 \\
\hline
\end{tabular}

analysis highlight the continued controversy on the optimal timing of SCPA while offering insight on the factors associated with surgical outcome.

\section{Mortality and Transplantation}

Similar to previous reports, deaths were relatively uncommon after SCPA. ${ }^{5,15}$ Compared with neonatal palliations, SCPA might result in less physiologic stress, is performed in some cases without cardiopulmonary bypass, and generally results in more favorable postoperative hemodynamics. It is also possible that the highest risk subjects die before undergoing SCPA. ${ }^{11}$

\section{Subject and SCPA Hospitalization Characteristics}

With the exception of the proportion of subjects undergoing aortopulmonary shunt takedown, no differences in subject or SCPA hospitalization characteristics were noted across the SCPA subtypes. Although the results of subgroup comparisons should be interpreted cautiously, given the small number of subjects for some groups, these results did not identify significant differences in subjects undergoing bilateral SCPA ${ }^{16}$ or a Kawashima procedure. ${ }^{17,18}$

\section{Age at SCPA}

The present analysis identified subject characteristics or events that might result in younger age at SCPA. The timerelated occurrences of interstage cardiac and pulmonary adverse events were associated with earlier SCPA. Consistent with previous reports, events such as residual arch obstruction $^{19}$ or narrowing of the aortopulmonary shunt ${ }^{20}$ likely trigger earlier SCPA to address specific causes of the adverse event and reduce the negative effect on single ventricle physiology. In contrast, the results did not confirm previously reported significant associations between age at SCPA and several factors considered potentially clinically relevant such as type of SCPA, oxygen saturation, presence of valve regurgitation, ${ }^{15}$ decreased ventricular ejection fraction, and increased ventricular end-diastolic pressure. Subjects who were younger at neonatal palliation or a Norwood operation might undergo SCPA sooner owing to concerns of volume overload from accessory pulmonary blood flow or the risk of interstage death. The independent association of race and timing of SCPA is difficult to interpret using variables derived solely from this data set. Largely a social construct, race is used in health research as a surrogate for many unmeasured environmental and biologic differences between individuals. Details of these factors were not available. However, the observation is not entirely surprising, because racial disparities have been described in almost every area of healthcare, including congenital heart disease. ${ }^{21}$

The timing of SCPA is a decision that balances clinical driving forces (eg, hypoxemia), previous operative results, and anticipated benefits of altered physiology. However, timing can also be influenced by the logistical challenges of busy clinical programs, such as bed space, operating room time, and physician availability. The interpretation of this balance is likely different among centers, as evidenced by the observation that younger age was independently associated with center after adjusting for measured subject factors. Similar center differences in surgical timing have been reported in the single ventricle population. ${ }^{22}$ These results highlight the lack of consensus regarding the optimal timing of the SCPA in infants with a single ventricle.

\section{LOS After SCPA}

Several of the factors associated with LOS could be considered more generalized measures of subject well-being before SCPA. In particular, the weight-for-age $z$ score, which was chosen as the primary endpoint for the main trial, reflects broadly on overall health in infants. Growth variables have previously been associated with single ventricle outcomes. $^{1,22,23}$ Ventricular end-systolic volume was 1 of several echocardiographic measures included in the statistical analyses and might represent a surrogate for heart failure. 
The number of days of ventilator use at the first neonatal surgery could represent underlying subject characteristics that complicate cardiothoracic surgery or some degree of perioperative lung injury that subsequently complicates SCPA. Finally, although the reasons that a subject undergoes an earlier SCPA are complex, younger age at SCPA was associated with a longer hospital stay even when adjusting for these available factors. Given the ongoing debate about optimal timing of SCPA, ${ }^{3,6}$ this observation is notable. Although the benefits of post-SCPA physiology can include favorable ventricular remodeling, less severe heart failure with improved growth, and avoidance of interstage death, it is possible that the potential benefits of elective SCPA at a younger age might be offset by potential complications related to a complex post-SCPA clinical course.

\section{Other Clinical Outcomes After SCPA—Unanticipated Interventions, Mechanical Ventilation, Chest Tube Duration}

Analysis of unanticipated postoperative procedures provides additional insight on the nature of the complications that occur in association with SCPA. The most common procedure in the present study was placement of a thoracostomy tube, and the remaining procedures were quite varied. For this reason, the multivariate model should be interpreted conservatively and reflects the generalized risk of unanticipated procedures rather than the risk of any specific event or procedure.

The binary outcomes for mechanical ventilator duration ( $>2$ days) and chest tube duration ( $>5$ days) were designed to identify predictors of outcomes considered suboptimal. The mean total duration for both variables was similar to that of previous reports. ${ }^{1,5}$ Younger age, along with elevated brain natriuretic peptide and multiple concurrent operative procedures, was predictive of a prolonged ventilator course. Worse ventricular function was predictive of chest tube drainage, which might be related to elevation in pulmonary venous pressure resulting from high ventricular filling pressures. Although direct catheter measurement did not support this hypothesis, the data from invasive measurements were frequently missing and the power could have been limited.

\section{Study Limitations}

The limitations of the present study included possible biases introduced by the original study cohort. Missing data, particularly hemodynamics from cardiac catheterization, limited the inclusion of some variables in multivariate modeling. Adverse events were only collected after the subject enrolled in the trial. For subjects who were enrolled in the trial after discharge from the neonatal hospitalization, events that might have occurred after discharge and before enrollment were not captured in these analyses. The socioeconomic data were insufficient to aid in interpretation of race findings in the age at SCPA model. Finally, although this was a relatively large cohort of single ventricle subjects, the low prevalence of suboptimal SCPA outcomes might have result in limited power to detect associations in the multivariate model results listed in Table 4.

\section{CONCLUSIONS}

This large multicenter cohort of infants with single ventricle identified factors associated with timing of SCPA and surgical outcome. The incidence of death or heart transplantation was $5 \%$ by 14 months of age. Younger age at SCPA was associated with case complexity and interim morbidities. In addition, significant practice variation in the age at SCPA among centers, independent of subject factors, highlighted the controversy regarding the optimal timing of SCPA. Factors associated with LOS and other measures of operative morbidity might offer insights for improving pre-SCPA care and surgical outcome. The risks and benefits of elective SCPA at younger age require additional study.

\section{References}

1. Anderson JB, Beekman RH III, Border WL, Kalkwarf HJ, Khoury PR, Uzark K, et al. Lower weight-for-age z-score adversely affects hospital length of stay after the bidirectional Glenn procedure in 100 infants with a single ventricle. J Thorac Cardiovasc Surg. 2009;138:397-404.e1.

2. Ashburn DA, Blackstone EH, Wells WJ, Jonas RA, Pigula FA, Manning PB, et al. Determinants of mortality and type of repair in neonates with pulmonary atresia and intact ventricular septum. J Thorac Cardiovasc Surg. 2004;127:1000-8.

3. Ghanayem NS, Tweddell JS, Hoffman GM, Mussatto K, Jaquiss RD. Optimal timing of the second stage of palliation for hypoplastic left heart syndrome facilitated through home monitoring, and the results of early cavopulmonary anastomosis. Cardiol Young. 2006;16(Suppl 1):61-6.

4. Jaquiss RD, Siehr SL, Ghanayem NS, Hoffman GM, Fedderly RT, Cava JR, et al. Early cavopulmonary anastomosis after Norwood procedure results in excellent Fontan outcome. Ann Thorac Surg. 2006;82:1260-6.

5. Kogon BE, Plattner C, Leong T, Simsic J, Kirshbom PM, Kanter KR. The bidirectional Glenn operation: a risk factor analysis for morbidity and mortality. J Thorac Cardiovasc Surg. 2008;136:1237-42.

6. Petrucci O, Khoury PR, Manning PB, Eghtesady P. Outcomes of the bidirectional Glenn procedure in patients less than 3 months of age. J Thorac Cardiovasc Surg. 2010;139:562-8.

7. Slavik Z, Lamb RK, Webber SA, Devlin AM, Keeton BR, Monro JL, et al. Bidirectional superior cavopulmonary anastomosis: how young is too young? Heart. 1996;75:78-82.

8. Kaltman JR, Schramm C, Pearson GD. The National Heart, Lung, and Blood Institute Bench to Bassinet Program: a new paradigm for translational research. J Am Coll Cardiol. 2010;55:1262-5.

9. Wernovsky G, Ghanayem N, Ohye RG, Bacha EA, Jacobs JP, Gaynor JW, et al. Hypoplastic left heart syndrome: consensus and controversies in 2007. Cardiol Young. 2007;17(Suppl 2):75-86.

10. Ohye RG, Goldberg CS, Donohue J, Hirsch JC, Gaies M, Jacobs ML, et al. The quest to optimize neurodevelopmental outcomes in neonatal arch reconstruction: the perfusion techniques we use and why we believe in them. $J$ Thorac Cardiovasc Surg. 2009;137:803-6.

11. Hsu DT, Zak V, Mahony L, Sleeper LA, Atz AM, Levine JC, et al. Enalapril in infants with single ventricle: results of a multicenter randomized trial. Circulation. 2010;122:333-40.

12. Hsu DT, Mital S, Ravishankar C, Margossian R, Li JS, Sleeper LA, et al. Rationale and design of a trial of angiotensin-converting enzyme inhibition in infants with single ventricle. Am Heart J. 2009;157:37-45.

13. US National Cancer Institute. Common terminology criteria for adverse events (CTCAE), revised edition. Bethesda, MD: US Dept. of Health and Human Services, National Institutes of Health, National Cancer Institute; 2009.

14. Sluysmans T, Colan SD. Theoretical and empirical derivation of cardiovascular allometric relationships in children. J Appl Physiol. 2005;99:445-57. 
15. Scheurer MA, Hill EG, Vasuki N, Maurer S, Graham EM, Bandisode V, et al. Survival after bidirectional cavopulmonary anastomosis: analysis of preoperative risk factors. J Thorac Cardiovasc Surg. 2007;134:82-9. 9e1-2.

16. Iyer GK, Van Arsdell GS, Dicke FP, McCrindle BW, Coles JG, Williams WG. Are bilateral superior vena cavae a risk factor for single ventricle palliation? Ann Thorac Surg. 2000;70:711-6.

17. Brown JW, Ruzmetov M, Vijay P, Rodefeld MD, Turrentine MW. Pulmonary arteriovenous malformations in children after the Kawashima operation. Ann Thorac Surg. 2005;80:1592-6.

18. Nath DS, Carden AJ, Nussbaum DP, Shin AJ, Khemani RG, Starnes VA, et al. Can the Kawashima procedure be performed in younger patients? Ann Thorac Surg. 2009;88:581-7.

19. Chessa M, Dindar A, Vettukattil JJ, Stumper O, Wright JG, Silove ED, et al. Balloon angioplasty in infants with aortic obstruction after the modified stage I Norwood procedure. Am Heart J. 2000;140:227-31.

20. Wang JK, Wu MH, Chang CI, Chiu IS, Lue HC. Balloon angioplasty for obstructed modified systemic-pulmonary artery shunts and pulmonary artery stenoses. J Am Coll Cardiol. 2001;37:940-7.

21. Nembhard WN, Salemi JL, Ethen MK, Fixler DE, Dimaggio A, Canfield MA. Racial/ethnic disparities in risk of early childhood mortality among children with congenital heart defects. Pediatrics. 2011;127:e1128-38.

22. Wallace MC, Jaggers J, Li JS, Jacobs ML, Jacobs JP, Benjamin DK, et al. Center variation in patient age and weight at Fontan operation and impact on postoperative outcomes. Ann Thorac Surg. 2011;91:1445-52.

23. Anderson JB, Kalkwarf HJ, Kehl JE, Eghtesady P, Marino BS. Low weight-forage z-score and infection risk after the Fontan procedure. Ann Thorac Surg. 2011; 91:1460-6.

\section{APPENDIX 1. INFANT SINGLE VENTRICLE TRIAL PARTICIPANTS}

National Heart, Lung, and Blood Institute: Gail Pearson, Victoria Pemberton, Mario Stylianou, Marsha Mathis*

Network Chair: Lynn Mahony, University of Texas Southwestern Medical Center, Dallas, Tex

Data Coordinating Center: New England Research Institutes, Lynn Sleeper (primary investigator [PI]), Steven Colan, Lisa Virzi,* Lisa Wruck,* Victor Zak, David F. Teitel

Core Clinical Site Investigators: Children's Hospital Boston, Jane W. Newburger (PI), Roger Breitbart, Jami Levine, Ellen McGrath, Carolyn Dunbar-Masterson; Children's Hospital of New York, Daphne Hsu* (study chair), William Hellenbrand (PI), Ashwin Prakash,* Seema Mital,* Darlene Servedio*; Children's Hospital of Philadelphia, Victoria L. Vetter (PI), Chitra Ravishankar, Sarah Tabbutt,* Meryl Cohen, Katherine Lee, Marisa Nolan, Stephanie Piacentino, Michelle Toms; Cincinnati Children's Medical Center, D. Woodrow Benson (PI), Catherine Dent Krawczeski, Lois Bogenschutz, Teresa Barnard, Steven Schwartz, ${ }^{*}$ David Nelson; North Carolina Consortium: Duke University, East Carolina University, Wake Forest University, Page A. W. Anderson (PI)—deceased, Jennifer Li (PI), Wesley Covitz, Kari Crawford,* Michael Hines, James Jaggers,* Theodore Koutlas, Charlie Sang, Jr, Lori Jo Sutton, Mingfen Xu; Medical University of South Carolina, J. Philip Saul (PI), Andrew Atz, Girish Shirali, Eric M. Graham, Teresa Atz; Primary Children's Medical Center and the University of Utah, Salt Lake City, Utah, L. LuAnn Minich (PI), John A. Hawkins-

\footnotetext{
* No longer at the institution listed.
}

deceased, Richard V. Williams, Linda M. Lambert, Marian E. Shearrow; Hospital for Sick Children, Toronto, Ontario, Canada, Brian McCrindle (PI), Elizabeth Radojewski, Nancy Slater, Svetlana Khaikin, Susan McIntyre.

Auxiliary Sites: Children's Hospital of Wisconsin, Nancy Ghanayem, Kathy Mussatto, Michele Frommelt, Lisa Young-Borkowski; University of Michigan, Albert Rocchini, Laurie Rodgers-Augustyniak, Echocardiography Core Laboratory: Children's Hospital Boston: Steven Colan, Renee Margossian

Genetics Core Laboratory: Children's Hospital of New York, Wendy Chung, Liyong Deng, Patricia Lanzano

Protocol Review Committee: Michael Artman, Chair; Judith Massicot-Fisher, Executive Secretary; Timothy Feltes, Julie Johnson, Thomas Klitzner, Jeffrey Krischer, G. Paul Matherne

Data and Safety Monitoring Board: John Kugler, Chair; Rae-Ellen Kavey, Executive Secretary; David J. Driscoll, Mark Galantowicz, Sally A. Hunsberger, Thomas J. Knight, Holly Taylor, Catherine L. Webb

\section{APPENDIX 2. LIST OF PREDICTORS EXPLORED}

The following list presents all the potential predictors that were explored. Tertiles and median cutoffs were included in the logistic regression analyses to understand any possible nonlinear association. In addition, when associations appeared nonlinear according to the tertiles, the lowest tertile or greatest tertile might have been analyzed as a dichotomous predictor. In the analysis of the length of stay, the associations of predictors with the outcome were explored using the generalized additive model regressions and plots.

\section{Treatment}

NITT enalapril (angiotensin-converting enzyme inhibitor) at pre-SCPA visit

Percentage of time taking enalapril or angiotensinconverting enzyme inhibitor

\section{Demographics and baseline characteristics}

Birth weight $(\mathrm{kg})$ (observed, tertiles, log transformed, square root transformed, $<2500 \mathrm{~g}$ )

Gestational age (observed, $<37$ vs $\geq 37$ weeks)

Gender

Race parent's highest level of education ( $1 d f$ and $5 d f)$

Annual household income ( $1 d f$ and $4 d f)$

Ventricular morphology (left vs right and mixed)

Systemic ventricular dysfunction at baseline

\section{Details of neonatal palliative surgery}

Age at surgery

Circulatory arrest time (observed, tertiles, above/below median)

Length of time ventilator required (observed, tertiles, above/below median, square root transformed)

Number of interventional catheterizations during neonatal hospitalization after palliative surgery 
Number of other surgeries after palliative surgery (collapsed to "any other surgeries")

\section{Pre-SCPA measures}

Pre-SCPA weight-for-age $z$ score (observed, $<-2$ vs $\geq-2$, $<-1$ vs $\geq-1$, tertiles)

Pre-SCPA oxygen saturation (observed, tertiles, above/ below median)

Pre-SCPA brain natriuretic peptide (observed, tertiles, low brain natriuretic peptide, log transformed)

\section{Pre-SCPA catheterization}

Mean systemic venous atrial pressure (observed, tertiles, above/below median)

Pulmonary capillary wedge pressure (observed, tertiles, above/below median)

End-diastolic pressure (observed, tertiles, above/below median)

Mean pulmonary artery pressure (observed, tertiles, above/below median)

Transpulmonary gradient (observed, tertiles, above/ below median)

Number of interventional procedures during the catheterization

\section{Pre-SCPA echocardiogram}

Overall atrioventricular valve regurgitation

Ejection fraction (observed, $>55 \%$ vs $\leq 55 \%, z$ score)

Mass/volume ratio (observed, tertiles, above/below median)

Mass/volume ratio $z$ score (observed, tertiles, above/ below median)
Mass (observed, tertiles, above/below median)

Mass $z$ score (observed, tertiles, above/below median)

End-diastolic volume (observed, tertiles, above/below median)

End-diastolic volume $z$ score (observed, tertiles, above/ below median)

End systolic volume (observed, tertiles, above/below median)

End-systolic volume $z$ score (observed, tertiles, above/ below median)

\section{Pre-SCPA feeding}

Whether the subject was consuming solid food before SCPA (yes/no)

Total daily calories consumed (observed, tertiles, above/ below median, log transformed)

Total calories consumed per day per kilogram (observed, tertiles, above/below median, log transformed)

Pre-SCPA feeding type

Tube fed before SCPA (yes/no)

\section{Interstage adverse events}

First serious adverse event

First cardiac general or cardiac arrhythmia adverse event

First pulmonary adverse event

Details of SCPA

Age at SCPA (observed, tertiles, $<140$ days vs $\geq 140$ days) for SCPA outcomes

Type of SCPA (bidirectional Glenn, hemi-Fontan, bilateral bidirectional Glenn, Kawashima)

Number of cardiac procedures concurrent with SCPA 